\title{
Escuelas diferenciadas en España: un análisis cualitativo de las razones y percepciones de sus directivos
}

\section{Single-sex schools in Spain: a qualitative analysis of the reasoning and perceptions of their principals}

Dr. Jaume CAMPS BANSELL. Profesor Titular. Universitat Internacional de Catalunya (jaumecamps@uic.es). Dña. Elisabeth VIERHELLER. Doctoranda en la Facultad de Filosofía y Letras. Universidad Nacional de Cuyo, Argentina (elivierheller@gmail.com).

\section{Resumen:}

La educación diferenciada mantiene su presencia en el panorama escolar de España, en un entorno de coeducación generalizada. Aunque existen algunas investigaciones sobre las razones para mantener 0 promover este modelo escolar en los países anglosajones, no hay estudios para España. El objetivo propuesto es ahondar en las razones y motivaciones - pedagógicas u otras - de la creación o mantenimiento de la separación de los sexos en las escuelas diferenciadas en España, $\mathrm{y}$-en la percepción de los directivosconocer los resultados y buenas prácticas que llevan a cabo en sus escuelas. Se ha partido del análisis cualitativo de doce entrevistas en profundidad con directivos de esas escuelas, que se grabaron, transcribieron y procesaron con el programa Atlas.ti. El análisis de los resultados permitió llegar a diversas conclusiones: la separación es percibida como práctica potenciadora de los objetivos de la educación personalizada; la escolarización diferenciada, dentro de una planificación educativa adecuada, reporta ventajas para aspectos como la igualdad de género o la excelencia escolar; permite dar respuesta a una demanda de las familias; la inclusión de planes formativos para la igualdad en esas escuelas es una práctica generalizada. Se destaca también la consideración de la trascendencia educativa del profesorado como modelo de género para conseguir una mayor igualdad de oportunidades.

Descriptores: sexo, género, escuela masculina, escuela femenina, educación diferenciada.

\section{Abstract:}

Single sex education is still present in Spain's school panorama within a predominantly co-educational environment. Although there are some pieces of research from English-speaking countries on the reasons

Fecha de recepción de la versión definitiva de este artículo: 03-05-2017.

Cómo citar este artículo: Camps Bansell, J. y Vierheller, E. (2018). Escuelas diferenciadas en España: un análisis cualitativo de las razones y percepciones de sus directivos | Single-sex schools in Spain: A qualitative analysis of the reasoning and perceptions of their principals. Revista Española de Pedagogía, 76 (269), 101-117. doi: https:// doi.org/10.22550/REP76-1-2018-05 
for promoting or maintaining this model of schooling, there are no studies from Spain. The proposed objective of this research is to examine in depth the reasons and motivations - pedagogical and otherwise- for creating or maintaining separation by sexes in single-sex schools in Spain, and -in the principals' perceptions - discover the good practices used in these schools and their outcomes. This work is based on qualitative analysis of twelve in-depth interviews with principals of these schools, which were recorded, transcribed, and processed using the Atlas.ti program. The analysis of the results enabled us to reach a variety of conclusions: separation is viewed as a practice that favours the objectives of individualised education; single-sex schooling, within an appropriate educational plan, delivers advantages in areas such as gender equality or academic excellence; it makes it possible to meet a wish of the families; the inclusion of training plans for equality in these schools is a general practice. It is also apparent that the educational importance of teaching staff as gender role models to achieve greater equality of opportunities is valued.

Keywords: sex, gender, boys' schools, girls' schools, single sex education.

\section{Introducción}

La coeducación generalizada de niñas y niños en España contribuyó a eliminar muchas barreras sociales de género, posibilitando a hombres y mujeres explorar terrenos profesionales y vitales que durante siglos habían estado destinados exclusivamente al otro sexo; sin embargo, después de muchos años de experiencia de escuela mixta, se mantienen todavía diversas dificultades en el campo de la igualdad (Camps y Vidal, 2015; Duru-Bellat, 2010; UNESCO, 2012).

Estas dificultades han generado preocupación en los últimos decenios, y han llevado a la aparición de prácticas educativas nuevas (Consell Superior d'Avaluació del Sistema Educatiu, 2012), algunas de ellas dentro de la ya tradicional escuela coeducativa. Y otras, como la escuela diferenciada o la coeducación reflexiva, que proponen una separación de los sexos completa o parcial; especialmente en Estados Unidos, la progresiva aparición de nuevas escuelas públicas diferenciadas 0 de coeducación reflexiva motivó una creciente investigación en ese campo.

Es un hecho cada vez más reconocido que el entorno de aprendizaje es un factor importante en la motivación del alumnado, en sus resultados y en sus opciones de futuro (Hornstra, Mansfield, Van der Veen, Peetsma y Volman, 2015).

En este estudio, nos proponemos ahondar en el desarrollo de la escuela diferenciada en España; más en concreto, nos centraremos en las motivaciones y percepciones de las personas que desempeñan un cargo de dirección en esos centros educativos. Si bien empieza a existir un cuerpo de investigación que permite cierta comparación cuantitativa de resultados educativos entre esas prácticas escolares de género (en escuela mixta y diferencia- 
da), la investigación cualitativa en este campo es extremadamente reducida (Datnow y Hubbard, 2001; Mael et al., 2005). Posiblemente debido al debate generado en los últimos años sobre la mayor o menor efectividad de la escuela diferenciada, se ha tendido sobre todo a explorar el rendimiento escolar a partir de las variables sexo y modalidad escolar, sin profundizarse en las razones por las que se opta por esta tipología organizativa.

\section{Estado de la cuestión}

Los dos meta-análisis comparativos de resultados de la escuela mixta y diferenciada existentes (Mael et al., 2005; Pahlke, Hyde y Allison, 2014) prácticamente no inciden en aquello que nos proponemos en este estudio; al mismo tiempo, muestran un cuerpo de investigaciones centrado en el mundo anglosajón, difícilmente transferible a nuestro entorno. En Estados Unidos, hasta muy recientemente no ha habido una aproximación a la cuestión desde las motivaciones de las personas que toman esas decisiones (Fabes, Pahlke, Borders y Galligan, 2015), a pesar de la creciente popularidad que ha tenido este modelo en la escuela pública de ese país.

Nuestro propósito es llegar a una mayor y mejor comprensión de las razones por las que los directivos de las escuelas single-sex mantienen u optan por esta modalidad. Esclarecer estas cuestiones tiene sobrado interés, y más cuando el debate ha entrado en el mundo de las políticas educativas (Martínez López-Muñiz et al., 2015).

La escuela diferenciada se organiza en espacios de un solo sexo, como centro educativo o bien en sus aulas. En los países del sur de Europa y en Latinoamérica, esta modalidad escolar se ha fundado mayoritariamente en escuelas de "educación personalizada” (García-Hoz, 1977, 1989). Esta educación personalizada se puede definir como una visión y un conjunto de prácticas pedagógicas que intentan estimular al alumno a dirigir la propia vida, a desarrollar la capacidad de hacer efectiva la libertad personal, participando -con sus características peculiares - en la vida comunitaria; eso implica que, en la escuela, el alumno o la alumna es el protagonista del proceso educativo. De otra parte, la unicidad de la persona lleva a estas escuelas a considerar el aporte que cada alumno puede hacer a la comunidad, fomentándose la creatividad. El profesorado interviene como una ayuda o mediador en ese proceso (Carrasco, 2011).

La escuela diferenciada, dentro de la educación personalizada, separa a los niños y las niñas considerando que esa práctica educativa favorece las oportunidades de cada sexo, al desactivarse en las aulas las presiones de género, y reconociendo que el sexo es la principal categoría en la configuración psicosocial de un grupo de alumnos (Maccoby, 2003; Páez, 2004). Se ha descrito esa separación como potenciadora de la libertad de chicas y chicos, como facilitadora para el alumnado de la exploración de nuevos territorios vitales y académicos, evitando que los estereotipos y prejuicios de género frenen sus intereses (Camps et al., 2015; Duru-Bellat, 1995).

En los países anglosajones la escuela diferenciada está muy presente, tanto en la red pública como privada, fundamentándose en otra perspectiva -más prag- 
mática - enfocada a los resultados, y en ocasiones fundada en cierto biologismo esencialista de las diferencias de sexo; asimismo, el desarrollo de las oportunidades de las mujeres ha sido uno de los potenciadores de escuelas femeninas (Park, Behrman y Choi, 2013).

La generalización de la escuela mixta se dio, en los países occidentales, con rapidez y por motivos no propiamente educativos 0 de igualdad (Fize, 2003; Riordan, 1990; Sadker y Sadker, 1995). Un número de escuelas pequeño, pero no despreciable, mantuvieron la separación de los sexos; no han sido estudiadas las razones. Esas escuelas, por minoritarias, se vieron en la necesidad de mostrar su efectividad (Riordan, 1990) a través de estudios cuantitativos que la comparaban con la escuela coeducativa.

Los pocos estudios cualitativos existentes sobre la escuela diferenciada se han centrado principalmente en el plano competencial de los alumnos y alumnas (Pahlke et al., 2014; Shmurak, 1998; Streitmatter, 1999; Svartoien-Conway, 2000) 0 bien en los procesos de implementación de esas escuelas (Chadwell, 2010; Datnow, Hubbard y Woody, 2001; Datnow et al., 2002). Existen también algunas aproximaciones cualitativas que parten de las percepciones de los alumnos (Camps et al., 2015; Jackson, 2013).

Datnow et al. (2001) afirman que en la actualidad se valora la educación diferenciada como estrategia para mejorar la conducta en clase, eliminar distracciones y disminuir la presión de los pares. Este cambio de perspectiva, por lo menos en parte, fue originado por Gilligan
(1993), quien proponía unas girl-friendly schools que se adaptaran a las necesidades de ellas. De modo especial a partir de ese momento, se suceden las propuestas single-sex orientadas al empoderamiento de las chicas, al tiempo que empiezan a reconocerse las dificultades de los chicos.

Las investigaciones sobre las motivaciones de los directivos para llevar a cabo educación diferenciada se circunscriben al ámbito anglosajón. Datnow y otros (2001), en su estudio de escuelas de California, reconocen que para la mayor parte de directivos, las prácticas de coeducación reflexiva y de escolaridad diferenciada son un medio para afrontar las necesidades de los alumnos con dificultades, y no un fin en sí mismas; entre los educadores, es generalizada la opinión de que estas modalidades escolares contribuyen a mejorar la atención de los chicos y chicas, y al aumento de su autoestima; y reprochan a la falta de tiempo, medios y respaldo político, la posibilidad de implementarlas.

Para la mayoría de educadores, el hecho de separar los sexos manteniendo la misma calidad educativa es suficiente para garantizar la igualdad de oportunidades; sin embargo, los educadores no son conscientes de los sesgos de género en su trabajo, ni tampoco reciben ayuda para evitarlos. También se afirma que, en muchas ocasiones, el profesorado percibe que los espacios single-sex facilitan un trato franco y sincero con el alumnado, sobre sus preocupaciones y temas de interés, y favorece ofrecerles consejos (Datnow et al., 2001).

Pahlke et al. (2014) reconocen que quienes promueven e implementan escuelas 0 
clases diferenciadas suelen valorar las mayores posibilidades de aprendizaje de cada sexo, y el aumento de su interés por las materias. En su meta-análisis, presenta algunas categorías de conjeturas sobre esas prácticas escolares single-sex por parte de quienes las proponen.

En primer lugar, las de quienes suponen un mayor interés y desarrollo académicos de chicas y chicos en esas escuelas. Entre estos, se dan tres puntos de vista:

a) Aquellos que se centran en las diferencias bio-psicológicas referidas al aprendizaje (Gurian, 2010; James, 2007; Sax, 2005), los cuales inciden - por ejemplo- en las posibilidades de la separación para atenuar estereotipos y acercar a las chicas a la tecnología por medio de didácticas específicas.

b) Los que se aproximan al tema desde la perspectiva psicosocial y vislumbran los efectos negativos del sexismo en entornos coeducativos (Chaponière, 2010; Duru-Bellat, 2010).

c) Aquellos que partiendo de presupuestos biológicos y psicosociales reconocen una mayor efectividad de los entornos single-sex para las minorías 0 los alumnos en situaciones de desventaja debido a su entorno social (Hubbard y Datnow, 2005; Riordan, 2015).

En segundo lugar quienes, independientemente de la efectividad de la separación de los sexos, proponen hacer posible la elección de este tipo de educación para aquellas familias que la deseen (Chadwell, 2010; Liben, 2015; Martínez López-Muñiz, 2015).

Lingard y otros (2009) han descrito algunas motivaciones de directores de escuela para implementar clases diferenciadas: las problemáticas de conducta y las dificultades académicas de los chicos, evitando que eso impacte en los demás alumnos; y facilitar entornos en que los chicos se motiven para aquellas materias que los estereotipos sociales muestran como más adecuadas para las chicas. Estos directores relacionan esas dificultades con la falta de modelos masculinos entre el profesorado.

Para Fabes et al. (2015), faltan estudios que permitan comprender las razones del incremento de escuelas single-sex en Estados Unidos en los últimos años por parte de quienes toman esas decisiones. Los resultados de su investigación confirman que los directivos escolares con experiencia en esa modalidad acostumbran a tener una actitud más positiva hacia ella, la perciben como más efectiva, y -con mayor frecuencia que los directores de escuela coeducativa - utilizan argumentos esencialistas.

Más en concreto, y siempre referido a escuelas públicas de Estados Unidos, Fabes (2015) detectó, principalmente, en los directores, motivaciones de mejora académica del alumnado para el establecimiento de su escuela como single-sex; unos pocos directores aducían también razones de mejora del comportamiento, ligadas al mejor desarrollo académico de los estudiantes. También describió cómo bastantes directores indicaban la influencia de los titulares de la escuela para que fuera diferenciada por sexos, seguido del interés por parte del profesorado 0 de las familias del entorno. La profusión de libros, congresos y talleres sobre la escuela diferenciada en Estados Unidos también 
son citados como factores que ayudaron en la toma de la decisión. Algunos aspectos positivos citados frecuentemente por los directores, una vez transformada la escuela en single-sex, fueron la mejoría en la satisfacción de maestros y familias, en las relaciones entre alumnado y profesorado, y en el rendimiento académico. Un tercio de directores también comentaron que el cambio a escuela diferenciada había perjudicado algún aspecto, como por ejemplo el comportamiento en las clases de chicos. Algunos detectaron problemas organizativos debido al desequilibrio numérico de los sexos en algunos cursos.

Finalmente, se ha descrito la educación diferenciada como una práctica especialmente acorde con la llamada "educación personalizada", modalidad escolar teorizada inicialmente por García Hoz. La separación de los sexos supondría, al mismo tiempo, una adaptación al modo de ser persona mujer o persona hombre (Ahedo, 2012), y una facilidad para conseguir los objetivos educativos que caracterizan a la escuela personalizada (Camps et al., 2015).

En España, aunque sin estudios que lo confirmen, algunos autores insisten en que las razones de la escuela diferenciada se encuentran en la política, la ideología 0 en cuestiones religiosas (Bonal, 1997; Subirats, 2010).

El marco que hemos ofrecido hasta aquí nos puede ayudar a categorizar las cuestiones que se proponen en esta investigación. Sin embargo, estos estudios citados difícilmente son extrapolables a lo que ocurre en nuestro entorno, tanto por las diferencias socioculturales como por el distinto paradigma educativo.

\section{Preguntas de investigación}

En España existen aproximadamente doscientas escuelas diferenciadas (EASSE, 2016), todas ellas de iniciativa y titularidad privada. El debate -público y científico- y el desconocimiento que existe sobre esta modalidad escolar, nos han llevado a centrar nuestra atención en ella, dejando de lado las escuelas de coeducación reflexiva por su escaso número y la dificultad para reconocerlas como tales.

Más allá de efectividad académica y formativa, se pretende una investigación que dé respuesta a las siguientes cuestiones:

a) Cuáles son las razones y motivaciones - pedagógicas $u$ otras - de la creación o mantenimiento de la separación de los sexos en las escuelas diferenciadas en España.

b) Cuáles son -en la opinión de los directivos - los resultados y buenas prácticas que llevan a cabo las escuelas diferenciadas, desde una perspectiva de género y aprovechando la separación de niños y niñas.

\section{Metodología}

En este estudio se ha optado por una metodología cualitativa, la cual nos permite observar la realidad a través de los ojos de los participantes, entrar en la subjetividad de los agentes educativos. La revisión bibliográfica muestra que perseguimos unos objetivos no abordados anteriormente en el contexto español, por lo que las razones y motivaciones de los directores presentados y analizados pueden diferir notablemente respecto a lo que reportan otras investigaciones en este campo realizadas en contextos diferentes. 
La aproximación cualitativa supone así un primer acercamiento a una realidad inexplorada, y puede servir de base para futuros trabajos cuantitativos que permitan una generalización más fiable.

\subsection{Participantes}

Fueron entrevistados doce directores (ocho hombres y cuatro mujeres) de escuelas diferenciadas españolas. La edad media de los participantes fue de 48 años, en un rango de entre 40 y 61 años de edad. En promedio, tenían 13 años de experiencia en cargos directivos de escuela diferenciada, en un rango de entre 1 y 31 años. De las escuelas que dirigían los participantes, ocho eran masculinas y cuatro femeninas, con gran diversidad en los años de antigüedad de los centros (de 3 y 53 años) y en el perfil sociocultural medio de sus familias.

La selección de los participantes se realizó en dos de las comunidades autónomas españolas con más escuelas diferenciadas (Madrid y Cataluña); se obtuvieron los correos electrónicos de las escuelas o directores a través de las páginas web de los centros 0 de llamadas telefónicas. Se enviaron por correo electrónico - progresiva y aleatoriamente- mensajes solicitando la entrevista, exponiendo los objetivos y metodología del estudio, y finalmente concretando una cita con aquellos que respondían afirmativamente. Con la saturación teórica se detuvo el proceso de solicitudes de entrevista. De los diecisiete directivos a los que se llegó a solicitar entrevista, respondieron afirmativamente doce. Previamente a cada entrevista se entregaron de nuevo por escrito (correo electrónico) los objetivos del estudio, y se garantizó la confidencialidad de la iden- tidad del entrevistado; al mismo tiempo se les informó de la necesidad de registro digital de la entrevista para su posterior transcripción y análisis. Las entrevistas se realizaron de mayo a julio de 2016.

\subsection{Entrevistas}

Se desarrolló con cada participante una entrevista en profundidad semi-estructurada. Todas las entrevistas tuvieron lugar en sus respectivas escuelas, con una duración de entre 60 y 80 minutos, y las realizaron directamente los autores con los participantes.

$\mathrm{Al}$ inicio de la entrevista se preguntaban aquellos aspectos de contexto que podían ser relevantes para una mejor comprensión del resto de la conversación (el nivel socioeconómico medio de las familias del centro, si el entrevistado había participado como alumno o profesional en centros coeducativos, etc.).

Para establecer una secuenciación de los temas sobre los que establecer las preguntas de las entrevistas, se tomó el esquema de Wharton (2012). Esta autora propone abordar las cuestiones de género desde tres grandes marcos, sobre los que pivotan las interpretaciones de distintas situaciones sociales:

- El individual, que incluye las concepciones sobre las diferencias entre los sexos y su origen.

- El de la interacción, que aborda el género desde los contextos en los que los individuos interaccionan.

- El institucional, el cual reconoce que las interacciones en muchas ocasiones se dan en organizaciones, las cuales tienen un papel relevante en los 
roles, las posiciones y las expectativas que se dan sobre los individuos.

Partiendo de este esquema previo, el guion de los puntos a tratar en las entrevistas fue elaborado a partir de la revisión bibliográfica, y distribuido en temas y subtemas.

Para obtener el máximo de información, las preguntas establecidas se completaron con otras más específicas cuando la respuesta parecía escueta o poco comprensible («¿En qué aspectos lo ha observado?» «¿En qué edades se manifiesta especialmente?» Etc.).

Las entrevistas se realizaron en catalán 0 castellano dependiendo de la lengua materna 0 preferencia del participante y del entrevistador; se presentan todas las citas textuales en castellano, después de las convenientes traducciones.

\subsection{Análisis}

El análisis se ha llevado a cabo utilizando el programa informático Atlas.ti en su versión 7.5.10. A nivel textual, se han fragmentado los doce documentos primarios (transcripciones de las entrevistas) en citas que han sido compiladas con códigos previamente establecidos a partir de las dimensiones predefinidas, y algunos otros emergentes que han surgido durante el análisis. El corpus analizado contiene 630 citas y 33 códigos.

\section{Resultados}

\subsection{Sobre el proyecto educativo}

En todos los casos, los directores hablan de su escuela como institución con voluntad de servicio social y con principios cristianos, aunque la mayor parte niega una confesionalidad oficial. También hay unanimidad en presentar, como aspectos centrales del proyecto educativo, la educación personalizada y la importancia de las relaciones entre escuela y familias. Aparecen en diversas entrevistas el trato personalizado con el alumnado y sus familias, la educación socioemocional, la educación integral, la trascendencia de la persona, la educación en las nuevas tecnologías, la importancia de las lenguas y la educación en valores.

Al hablar sobre el ideario y el proyecto educativo, solamente tres directores hacen referencia a la educación diferenciada, presentándola al final como práctica especialmente acorde al proyecto escolar: «No es el principal rasgo de nuestra escuela» (entrevista 1).

A pesar de esa posición no central de la escolarización separada de los sexos en el proyecto educativo, diez de los directores afirman que es un aspecto organizativo al que no renunciarían fácilmente: «es una de nuestras señales de identidad [...]. Es una cuestión de demanda por parte de las familias» (entrevista 1). Dos de ellos, en escuelas de reciente creación con algunos cursos coeducativos por falta de suficientes alumnos para implementar una segunda línea, reconocen que tienen como objetivo la separación de los sexos por clases siempre que sea posible.

Preguntados sobre la coincidencia de la escolarización diferenciada y el ideario cristiano, diez de los doce entrevistados no aprecian relación alguna; los que dan más explicaciones, concretan que las escuelas 
privadas anteriores a la generalización de la coeducación tenían mayoritariamente ese ideario, por lo que les parece lógico que se mantenga la correlación entre esos dos aspectos; "si alguna vez ha habido esta concepción moral, lo que está claro es que en la actualidad no es en absoluto el fundamento de las prácticas de esta escuela (refiriéndose a la escolarización diferenciada)» (entrevista 2). De los dos que ven cierta posible relación, una directora comenta que quizás influya la consideración cristiana de la distinción entre hombre y mujer, aunque no lo presentan como motivo principal.

Todos afirman que el proyecto educativo de sus escuelas incluye aspectos de género; tanto la educación diferenciada, como aspectos de igualdad. Siete directores hicieron referencia explícita a la existencia en su escuela de un plan de formación sobre igualdad de género, específico 0 bien incluido en un plan de formación general.

En tres entrevistas aparece el concepto de artificialidad de la escuela. Con este término 0 con otros, once de los doce entrevistados hacen alguna referencia a que la escuela no puede ni debe reflejar la sociedad: "la misión de la escuela no es reflejar la sociedad. La escuela debe cambiar a las personas que cambiarán el mundo» (entrevista 2); muchos de ellos argumentan que esa artificialidad está necesariamente presente en la escuela en aspectos como separar por edades, etc.; 0 bien por la necesidad de distanciarse de los aspectos sociales negativos. Y algunos añaden que precisamente en esa artificialidad se encuentran algunas potencialidades de los centros educativos.

\subsection{Desarrollo social y académico del alumnado}

En las percepciones de las directoras y directores sobre la socialización, no existe una visión negativa de la cooperación y complementariedad intersexual que se da en las aulas mixtas. Algunos entienden esa intersexualidad escolar como imprescindible en épocas en las que no existía mixticidad social.

Todos hablan de la inexistencia de efectos negativos insalvables de socialización en la separación escolar de chicas y chicos, puesto que sus entornos extraescolares son mayoritariamente mixtos: «tengo la experiencia de que todas nuestras alumnas viven en el mundo real; en el colegio pasan unas horas al día, pero eso se complementa con la vida familiar que tienen, con sus relaciones fuera del colegio... El colegio no es la exclusividad de su vida» (entrevista 9). Algunos reconocen que la separación impide, en la escuela, un aporte bidireccional o de complementariedad, aunque aprecian las ventajas de la separación y la eficacia de las estrategias empleadas para subsanar ese déficit. Todos hablan, basándose en la experiencia, de la naturalidad y normalidad de las relaciones vitales que mantiene su alumnado con el otro sexo fuera de la escuela 0 en su vida post-escolar; varios directivos afirman que las familias que les confían a sus hijos no lo habrían hecho si percibieran efectos negativos. Dos directores y una directora explican que han preguntado con cierta frecuencia a los alumnos mayores 0 antiguos alumnos si tienen dificultades para relacionarse con el otro sexo, y coinciden en que la respuesta ante esa suposición es la risa. 
En su valoración de la separación escolar de los sexos, todos consideran ciertas potencialidades de su modelo pedagógico que pueden convertirse en ventajas educativas. Dos aspectos se repiten en diversas entrevistas:

En primer lugar, se habla de la importancia de la consolidación de la identidad personal como imprescindible para la socialización; y presentan la separación como ventaja para ello: «al no tener a los chicos, una ventaja es que las alumnas crecen para tener una personalidad propia» (entrevista 8).

En segundo lugar, comentan la trascendencia psicosocial de la separación para crear un clima de naturalidad en las acciones, unas posibilidades expresivas libres de presiones de género, sin vergüenza, etc.; un director dice que muchos alumnos le han comentado que el hecho de que no haya chicas «hace que sea más yo mismo, y actúe con naturalidad» (entrevista 5); otro afirma que «permite un descubrimiento de la sexualidad más tranquilo... más sereno, con menos ensayo y error... También empodera a las chicas a descubrirse como chicas, su identidad, en no asociar que son mujeres y por tanto deben gustar y complacer a los demás» (entrevista 2). Todos los directores que hablan del tema afirman la facilidad de la escuela diferenciada para la educación afectivo-sexual, por la comodidad que supone para los alumnos tratar esos temas en aulas separadas.

Tres de los entrevistados hablan de la distracción que supone para el alumnado la presencia del otro sexo, a partir de cierta edad, lo que puede dificultar el aprendizaje 0 las amistades con otros del mismo sexo.
Han aparecido algunas explicaciones sobre acciones que se llevan a cabo para fomentar una socialización adecuada con el otro sexo. En diversas entrevistas aparece la tutoría individual, práctica común en las doce escuelas, como espacio de diálogo en el que se puede hablar de ello, y en el que se aconseja y orienta.

Preguntados los directores y directoras sobre la contribución de la escuela diferenciada a erradicar la violencia hacia las mujeres, de forma generalizada reconocen que sus escuelas contribuyen a la solución del problema, por fomentarse decididamente en sus centros el respeto al otro: «el hecho de que el trato con las chicas no sea $\tan$ frecuente y tan constante... hace que cuando se produce el encuentro sean más respetuosos» (entrevista 1); «las situaciones de violencia de los chicos hacia las chicas desaparecen» (entrevista 11). Sin embargo, no todos relacionan directamente ese respeto con la separación de los sexos en la escuela: «hay otras variables, que no tienen nada que ver con la educación mixta 0 diferenciada» (entrevista 4).

\subsection{Formación docente y prácticas es- pecíficas}

Sobre su formación específica para la educación diferenciada, todos los directores y directoras entrevistados -excepto dos - afirman haber leído diversos libros sobre la cuestión. Todos han leído artículos y asistido a congresos centrados en esta modalidad escolar. Sobre la formación que reciben sus profesores, todos confirman que algunas de las sesiones de formación previstas tratan sobre esta cuestión. 
Hay unanimidad en que no existe particularidad alguna en el currículo por el hecho de ser una escuela diferenciada. Sobre la existencia de específicas estrategias docentes para cada sexo, prácticamente todos responden negativamente. Sin embargo, también comentan que puede haber diferencias conscientes 0 inconscientes por el hecho de adaptar su tarea educativa al alumnado que tienen en clase, que es de un sexo concreto.

Sobre el perfil del profesorado, en diez de las doce escuelas representadas el sexo del profesorado coincide siempre con el de los alumnos en las etapas obligatorias. Todos reconocen cierta incidencia del sexo del profesor en el plano educativo, y relatan diversos aspectos positivos de la coincidencia de sexos profesor-alumno: se elimina la facilidad de que una escuela de chicos acabara con casi solo profesoras y la falta de modelos masculinos derivada; la mayor confianza que se genera entre el alumnado y el profesorado.

\subsection{Retos sociales y escolares de género}

Respecto a los retos sociales de género a los que la escuela puede y debe contribuir, casi todos se refieren a la igualdad desde diversas perspectivas: igualdad de oportunidades, erradicar el sexismo y la violencia de género, eliminar estereotipos, etc.

Sobre los estereotipos en relación a la separación, las respuestas coinciden en algunos aspectos como la ya comentada existencia en muchos de los centros de planes de formación para la igualdad. Por otra parte, diversos entrevistados señalan el efecto limitador de los estereotipos, y la ventaja de la separación para dar más libertad y oportunidades a cada sexo: «los estereotipos de género solo son negativos cuando encorsetan 0 limitan la libertad del individuo... Los estereotipos son las negaciones que se imponen a los hombres y las mujeres... No veo que la escuela diferenciada comporte una limitación de las posibilidades de elección, en el mundo académico 0 personal» (entrevista 2). Otro director comenta que «aquí hay chicos que participan en teatro, coro, hacen servicios a la comunidad, a los más pequeños en el comedor... y si hubiera chicas pensarían que es algo para chicas» (entrevista 5). Algunos directores y directoras concretan otras actividades dirigidas a combatir los estereotipos: «que tengamos enseñanza diferenciada no quiere decir que a las niñas les enseñamos a planchar y a los niños a arreglar enchufes; enseñamos a todos lo mismo, y aquí se enseña a los niños tareas domésticas y a las niñas labores de mantenimiento de la casa» (entrevista 6).

Entre las explicaciones de cómo la separación beneficia las oportunidades de chicas y chicos, de una parte destacan que la ausencia del otro sexo hace que cualquier tarea sea apropiada para el alumnado, independientemente de los estereotipos sociales: el hecho de ser un centro diferenciado "hace que (las chicas) se planteen que las ciencias es una asignatura más... igual que la educación física» (entrevista 8); diversos entrevistados hablan de las posibilidades de los entornos diferenciados, puesto que en ellos las asignaturas no son percibidas en clave de género, y son experimentadas con mayor naturalidad: «el condicional del sexo de algún modo desaparece, y únicamente 
queda el condicionante académico; y facilita que otras cosas - como el rol habitual de sexo- desaparezca, y cada uno pueda desarrollarse con más tranquilidad» (entrevista 11). Y de otra parte, la coincidencia de sexo con el profesorado establece modelos de su sexo para todos los ámbitos. Muchas entrevistas se refieren a las opciones postobligatorias de su alumnado, como poco condicionadas por los estereotipos sociales sobre lo adecuado para cada sexo (en comparación con la escolarización mixta): «al haber solo chicas, está probado que el acceso a ingenierías 0 carreras de ciencias se refuerza» (entrevista 10$)$.

\subsection{Ventajas e inconvenientes de am- bos modelos}

Preguntados sobre la coeducación, todos los directivos reconocen que la escuela mixta puede educar con efectividad; uno de ellos explicita que «la escuela mixta ha sido muy positiva en dar un primer paso en la igualdad de la mujer» (entrevista 8). Casi todos reconocen también que ambos modelos tienen ciertas ventajas e inconvenientes; y que la presencia de un solo sexo 0 de ambos genera diferencias en el clima del aula que merecen una atención pedagógica.

Entre las problemáticas de la coeducación que perciben, aparece en dos ocasiones la dificultad para reconocer y tratar las diferencias entre chicas y chicos por parte del profesorado. Otros dos directivos mencionan también las dificultades de socialización entre el alumnado en sus relaciones afectivas, que se rompen y recomponen con otros alumnos de las mis- mas aulas. Especialmente los que no han trabajado nunca en una escuela mixta, son muy prudentes en sus comentarios. En casi todos los casos, las explicaciones sobre las problemáticas de la coeducación son escuetas, algo superficiales y diferentes para cada entrevistado. Algunos directivos reconocen que es más sencillo educar en aulas de un solo sexo.

Entre las dificultades de la educación diferenciada, aparece en diversas entrevistas que las clases masculinas presentan un clima de mayor impulsividad y cierta indisciplina, mientras que las femeninas presentan con más frecuencia conflictos de tipo emocional y de competencia entre las alumnas; afirman -en muchos casos- que esas peculiaridades se resuelven con una gestión específica, y que no constituyen un problema.

Todos valoran las ventajas de la educación diferenciada, y no se plantean la posibilidad de convertirse en centros coeducativos excepto en un caso (por motivos organizativos de esa escuela concreta). Entre las ventajas, se destaca frecuentemente el potencial académico de la separación para reducir el fracaso escolar y su sesgo de género, creando un ambiente más académico y alejado de distracciones.

También todos reconocen la escuela diferenciada como beneficiosa para ambos sexos. Algunos añaden la facilidad que, tanto las aulas de chicos como las de chicas, suponen para el profesorado: «yo soy una persona de la escuela mixta. Cuando comencé a dar clase a chicos me di cuenta de la facilidad, de cómo esta distribución facilitaba la docencia y las prácticas docentes» (entrevista 2). 
Preguntados en concreto por las razones por las que su escuela es de educación diferenciada, se repiten en casi todas las entrevistas tres aspectos:

- La experiencia les ha mostrado que se trata de una práctica pedagógica que funciona: «nuestra experiencia en primaria y secundaria ha sido buena; ¿para qué vamos a cambiar?» (entrevista 12).

- Esa experiencia positiva de las clases single-sex se relaciona con el modelo pedagógico de la educación personalizada; la separación de los sexos es una aspecto más, que contribuye muy positivamente a los objetivos y la eficacia de ese modelo escolar: «hay un tema capital, y es que la educación diferenciada favorece la personalización de la educación» (entrevista 12).

- Se ofrece escuela diferenciada porque es la opción de los padres que la han elegido: «si no interesase a los padres, no habría ningún colegio diferenciado» (entrevista 6).

\subsection{Valoración de la escuela diferen- ciada por otros agentes}

Solo para alguna de las escuelas los directivos aprecian que sea la separación el principal motivo de las familias para optar por ese centro educativo. El hecho de haber estudiado en una escuela diferenciada y conocer su funcionamiento es otro motivo importante para buscar uno de esos centros para sus hijos. Otras familias, en ocasiones presentan dudas que se desvanecen cuando conocen la escuela. Para todos los entrevistados, los resultados educativos y el clima escolar son la gran motivación para las familias, aunque no los relacionen con la educación diferenciada.

Respecto al profesorado, los entrevistados son unánimes en afirmar que la inmensa mayoría de sus maestros valora muy positivamente la escuela diferenciada, mencionando algunos de ellos que esa valoración es compartida por los que habían trabajado en escuelas mixtas anteriormente.

En todos los aspectos descritos, no se aprecian diferencias significativas en función de si el entrevistado es hombre o mujer, o si la escuela es de chicas o de chicos.

\section{Conclusiones y discusión}

El primer objetivo de este estudio era ahondar en las razones y motivaciones de la creación 0 mantenimiento de escuelas diferenciadas en España. Parece confirmarse una estrecha relación entre la educación diferenciada y el modelo de escuela llamada de educación personalizada (Camps, 2015). Los directores coinciden en que, para las escuelas single-sex, la separación es una herramienta que se adapta a la pedagogía de la educación personalizada; y para ellos supone potenciar los objetivos de esta última, especialmente en lo que se refiere a la disminución de los estereotipos y la facilitación de la libertad y oportunidades del alumnado, de un modo semejante a como lo describe Lingard et al. (2009). En todas las entrevistas, los directores manifiestan la trascendencia de los aspectos de igualdad de género, y reconocen las posibilidades de la coeducación, pero aprecian las ventajas de la escolarización diferenciada recono- 
ciendo - a su vez- algunas desventajas de esta. Otros motivos que aparecen con frecuencia son las ventajas en resultados escolares, así como la creación de un ambiente más académico (Riordan, 2009) y de confianza entre los agentes escolares (Datnow et al., 2001). No se aprecia que los directivos centren su motivación por la escuela diferenciada en argumentos esencialistas sobre las diferencias entre los sexos, como sí parece que ocurre en otros países (Fabes et al., 2015), ni tampoco en razones ideológicas, políticas o religiosas (Subirats, 2010), sino más bien en razones pedagógicas y psicosociales.

Se puede observar también, de una parte, un componente pragmático en las explicaciones; la comprobación del buen funcionamiento de la escuela, que atribuyen en parte a la separación de los sexos, les lleva a no plantearse otras opciones organizativas (Riordan, 2008). Por otra parte, el factor de la elección de las familias aparece con frecuencia como una constatación del buen resultado, así como el ofrecer una opción a los padres que la deseen para sus hijos.

Por lo que respecta al segundo objetivo - percepciones sobre los resultados y buenas prácticas de la escuela diferenciada-, destaca la unanimidad entre los directores entrevistados. Por un lado, se valoran los resultados académicos, que se relacionan en parte con la separación de los sexos (Blakesley, 2013; Datnow et al., 2001); y se valoran los resultados personales de socialización, de eliminación de estereotipos y la consecución de una mayor igualdad de oportunidades en las elecciones post-obligatorias (Duru-Bellat, 1995; Riordan, 2009). Por otro lado, todas las escuelas representadas llevan a cabo formación específica del alumnado para potenciar la educación en la igualdad entre mujeres y hombres, y en el respeto mutuo. Para conseguir todo ello, los entrevistados comentan la formación que han recibido como directores, y que potencian entre el profesorado; especialmente entre los directores, existen diferencias significativas en su nivel de profundización y formación sobre las prácticas de educación diferenciada y los aspectos relacionados con la igualdad. Todos coinciden en que el currículo es idéntico en las escuelas de chicas y de chicos, aunque con variaciones didácticas por parte del profesorado que responden a la lógica adaptación al alumnado que tienen en clase, una de cuyas características es la coincidencia de sexo. También aparece con cierta frecuencia la necesidad de una gestión específica de las aulas diferenciadas de chicos para regular y aprovechar su mayor impulsividad en su propio beneficio (Blakesley, 2013); sin embargo, a diferencia de lo que describe Lingard et al. (2009), no hablan de la separación como una solución a los problemas comportamentales de los chicos.

Las facilidades de socialización que aportan las aulas single-sex - naturalidad en las relaciones, mayores posibilidades expresivas, etc. - que los entrevistados describen, coinciden con la investigación de Blakesley (2013) realizada en Canadá.

Existe unanimidad en reconocer aspectos positivos en la coincidencia de sexos profesor-alumno (Park et al., 2013).

Aunque con este estudio no podemos conocer la evolución en el tiempo de las percepciones de los directivos, sí se apre- 
cia en ellos una generalizada actitud positiva hacia las aulas single-sex, tal como las reseñan Fabes et al. (2015) para Estados Unidos.

Mientras que Hubbard et al. (2005) y Riordan (2015) describen con claridad las mayores ventajas de la escuela diferenciada para el alumnado en riesgo y en situaciones sociales desfavorecidas, no existe unanimidad entre los entrevistados sobre un determinado perfil de alumno para el que la educación diferenciada suponga especiales beneficios. Esto podría ser debido a que cada directivo conoce básicamente su propia escuela, con un perfil de alumnado concreto, y puede resultarles difícil establecer comparaciones.

Los directivos entrevistados reconocen las diferencias entre los sexos como un aspecto a tener en cuenta -aunque con poca atención a las neurociencias-, pero con frecuencia terminan su argumentación con referencias a la posibilidad de elección de estilos pedagógicos por parte de la familia, tal como afirma en su estudio Liben (2015).

No se han podido establecer diferencias significativas en función del sexo del entrevistado, 0 del hecho que la escuela fuera masculina o femenina (Pahlke, Bigler y Patterson, 2014).

De acuerdo con los resultados obtenidos, parece que las escuelas diferenciadas españolas analizadas se corresponden con los modelos heurísticos 3 y 5 presentados por el Consell Superior d'Avaluació del Sistema Educatiu (2012).

El principal aporte de este estudio es la exploración de las razones de los direc- tivos de escuelas diferenciadas de España para crear 0 conservar centros con esta práctica organizativa, tema hasta el momento solo investigado en países anglosajones. Como prospectiva, se sugiere una mayor profundización en este tema de estudio desde las perspectivas del profesorado, del alumnado y de las familias, aspectos prácticamente inéditos en España.

\section{Referencias bibliográficas}

Ahedo, J. (2012). Fundamento antropológico de la separación escolar de chicos y chicas. En E. Vierheller (Coord.), Nuevo paradigma escolar (pp. 39-60). Rosario: Logos.

Blakesley, S. (2013). Single-sex Education in Northern Canada: A Case Study of Trapline Elementary School. The Qualitative Report, 18, 1-14.

Bonal, X. (1997). Las actitudes del profesorado ante la coeducación. Propuestas de intervención. Barcelona: Graó.

Camps, J. (2015). Inteligencia de género para la escuela. Pamplona: Círculo Rojo.

Camps, J. y Vidal, E. (2015). Marte y Venus en el aula: las percepciones del alumnado sobre los efectos psicosociales de la escolarización mixta y diferenciada. revista española de pedagogía, 73 (260), 53-71.

Carrasco, J. B. (2011). Educación personalizada: principios, técnicas y recursos. Madrid: Síntesis. Chadwell, D. (2010). A gendered Choice. Thousand Oaks, CA: Corwin.

Chaponière, M. (2010). La mixité, une évidence trompeuse. Revue Française de Pédagogie, 171, 69-75.

Consell Superior d'Avaluació del Sistema educatiu (2012). Diferències en els resultats educatius de nois i noies a Catalunya. Barcelona: CSDA. Datnow, A., Hubbard, L. y Woody, E. (2001). Is Single Gender Schooling Viable in the Public Sector? Lessons from California's Pilot Pro- 
gram. Final Report. Recuperado de http://files. eric.ed.gov/fulltext/ED471051.pdf (Consultado el 12-5-2016).

Datnow, A. y Hubbard, L. (Eds.) (2002). Gender in Policy and Practice. Perspectives on Single-Sex and Coeducational Schooling. New York: Routledge-Falmer.

Duru-Bellat, M. (1995). Filles et garçons à l'école, approches sociologiques et psycho-sociales. Revue Française de Pédagogie, 110 (1), 75-109.

Duru-Bellat, M. (2010). La mixité à l'école et dans la vie, une thématique aux enjeux scientifques forts et ouverts. Revue Française de Pédagogie, 171, 9-13.

Fabes, R. A., Pahlke, E. Borders, A. Z. y Galligan, K. (2015). US principals' attitudes about and experiences with single-sex schooling. Educational Studies, 41 (3), 293-311.

Fize, M. (2003). Les pièges de la mixité scolaire. Paris: Presses de la Renaissance.

García Hoz, V. (1977). La educación personaliza$d a$. Valladolid: Miñón.

García Hoz, V. (Dir.) (1989). Tratado de educación personalizada. El concepto de persona. Madrid: Rialp.

Gilligan, C. (1993). In a Different Voice: Psychological Theory and Women's Development. Cambridge, Massachusetts: Harvard University Press.

Gurian, M. (2010). Boys and Girls Learn Differently! San Francisco, CA: Jossey-Bass.

Hornstra, L., Mansfield, C., Van der Veen, I., Peetsma, T. y Volman, M. (2015). Motivational teacher strategies: the role of beliefs and contextual factors, Learning Environments Research, 18 (3), 363-392. doi: 10.1007/s10984015-9189-y

Hubbard, L. y Datnow, A. (2005). Do single-sex schools improve the education of low-income and minority students? An investigation of California's public single-gender academies. Anthropology and Education Quarterly, 36 (2), 115-131.
Jackson, C. (2013). Can Single-sex Classes in Co-educational Schools Enhance the Learning Experiences of Girls and/or Boys? An Exploration of Pupils' Perceptions. British Educational Research Journal, 28 (1), 37-48.

James, A. N. (2007). Teaching the Male Brain. Thousand Oaks, CA: Corwin Press.

Liben, L. S. (2015). Probability Values and Human Values in Evaluating Single-Sex Education. Sex Roles, 72 (9), 401-426.

Lingard, B., Martino, W. y Mills, M. (2009). Single-Sex Classes and Schools for Boys. En B. Lingard et al. (Coords.), Boys and Schooling (pp. 86-118). New York: Palgrave MacMillan.

Maccoby, E. E. (2003). The Two Sexes. Growing up Apart, Coming Together. Cambridge: Harvard University Press.

Mael, F. et al. (2005). Single-Sex Versus Coeducational Schooling: A Systematic Review. Washington: Policy and Program Studies Service, U.S. Department of Education, Office of Planning, Evaluation and Policy Development.

Martínez López-Muñiz, J. L. et al. (2015). Legitimidad de los colegios de un solo sexo y su derecho a concierto en condiciones de igualdad. Madrid: Iustel.

Páez, D. (2004). Psicología social, cultura y educación. Madrid: Pearson.

Pahlke, E., Bigler, R. S. y Patterson, M. M. (2014). Reasoning About Single-Sex Schooling for Girls Among Students, Parents, and Teachers. Sex Roles, 71 (5), 261-271.

Pahlke, E., Hyde, J. S. y Allison, C. M. (2014). The Effects of Single-Sex Compared With Coeducational Schooling on Students' Performance and Attitudes, a Meta-Analysis. Psychological Bulletin, 140 (4), 1042-1072.

Park, H., Behrman, J. R. y Choi, J. (2013). Casual Effects of Single-Sex Schools on College Entrance Exams and College Attendance: Random Assignment in Seoul High Schools. Demography, 50 (2), 447-469. 
Riordan, C. (1990). Girls and boys in school. Together or separate? New York: Teachers College Press.

Riordan, C. (Dir.) (2008). Early Implementation of Public Single-Sex Schools: Perceptions and Characteristics. Washington, D. C.: US Department of Education.

Riordan, C. (2009). The Effects of Single-Sex Schools. En II Congreso Latinoamericano de Educación Diferenciada: Nuevos escenarios para la educación de mujeres y varones (pp. 98-131). Buenos Aires: ALCED Argentina. Riordan, C. (2015). Single-Sex Schools. A Place to Learn. Lanham, MD: Rowman \& Littlefield.

Sadker, M. y Sadker, D. (1995). Failing at Fairness. New York: Touchstone.

Sax, L. (2005). Why Gender Matters. New York: Doubleday.

Shmurak, C. B. (1998). Voices of Hope: Adolescent Girls at Single Sex and Coeducational Schools. New York: Peter Lang.

Streitmatter, J. L. (1999). For girls only: Making a case for single-sex schooling. Albany, NY: Sunny Press.

Subirats, M. (2010). ¿Coeducación o escuela segregada? Un viejo y persistente debate. Revista de la Asociación de Sociología de la Educación, 3 (1), 143-158.
Svartoien-Conway, J. I. (2000). In search of connection: How graduates from all-girls high schools describe their leadership involvement at a coeducational university. Universidad de Nebraska - Lincoln, USA.

UNESCO (2012). Atlas mundial de la igualdad de género en la educación. París: UNESCO.

Wharton, A. S. (2012). The Sociology of Gender. Oxford: Wiley-Blackwell.

\section{Biografía de los autores}

Jaume Camps es Profesor Titular de Teorías e Instituciones Educativas en el Departamento de Educación en la Universitat Internacional de Catalunya. Sus principales líneas de investigación se centran en las cuestiones de género en la escuela y en el desarrollo de la Educación Personalizada.

Elisabeth Vierheller es Vicepresidenta de la Asociación Latinoamericana de Centros de Educación Diferenciada Argentina. Tutora online y profesora de Educación Diferenciada. Doctoranda por la Universidad Nacional de Cuyo. Su principal línea de investigación es la educación diferenciada o single-sex en contextos sociales complejos. 


\section{revista española de pedagogía año LXXVI, nº 269, enero-abril 2018 \\ Spanish Journal of Pedagogy \\ year LXXVI, n. 269, January-April 2018 \\ sumario *}

\section{table of contents **}

José Antonio lbáñez-Martín

75 años al servicio de la educación

75 years at the service of education

\section{Estudios}

\section{Studies}

David Menéndez Álvarez-Hevia

Aproximación crítica a la Inteligencia Emocional

como discurso dominante en el ámbito educativo

A critical approach to Emotional Intelligence

as a dominant discourse in the field of education

Javier Tourón, Deborah Martín,

Enrique Navarro Asencio, Silvia Pradas

y Victoria Î́nigo

Validación de constructo de un instrumento

para medir la competencia digital docente

de los profesores (CDD)

Construct validation of a questionnaire

to measure teachers' digital competence (TDC)
Jordi Longás Mayayo, Elena Carrillo Álvarez,

Albert Fornieles Deu y Jordi Riera i Romaní

3 Desarrollo y validación del cuestionario

sobre condicionantes de éxito escolar

en alumnos de secundaria

Development and validation of a questionnaire

about determinants of academic success

in secondary school students

7 Notas

Notes

Carmen Caro Samada, Josu Ahedo Ruiz

y Francisco Esteban Bara

La propuesta de educación moral

de Kohlberg y su legado en la universidad:

actualidad y prospectiva

Kohlberg's moral education proposal and

25 its legacy at university: present and future

\footnotetext{
* Todos los artículos están publicados en inglés en la página web de la revista: www.revistadepedagogia.org.

** All the articles are published in English on the web page of the journal: www.revistadepedagogia.org.
} 


\section{Jaume Camps Bansell y Elisabeth Vierheller}

Escuelas diferenciadas en España:

un análisis cualitativo de las razones

y percepciones de sus directivos

Single-sex schools in Spain: a qualitative analysis

of the reasoning and perceptions of their principals

\section{Pilar Martínez Clares y Cristina González Lorente}

Orientación, empleabilidad e inserción laboral

en la universidad a través de un

Modelo de Ecuaciones Estructurales

Career guidance, employability, and entering

the workforce at University through a Structural

Equation Model

\section{Olga Duarte Piña}

La Enseñanza de la Historia: innovación y continuidad desde Rafael Altamira

Teaching History: innovation and continuity

since Rafael Altamira

141

Josefina Sánchez Rodríguez,

Talia Cristina Morillo Lesme y

\section{Concepción Riera Quintana}

Evaluación de las necesidades afectivas en niñas y niños adoptados: manifestaciones en su expresividad psicomotriz

Evaluating the affective needs of adopted children: demonstrations of psychomotor expressiveness

101

\section{Reseñas bibliográficas}

Martínez, M., Esteban, F., Jover, G. y Payá, M.

La educación, en teoría (Juan Luis Fuentes).

Sarramona, J. Conservadores e izquierdistas frente a la educación (José Antonio Jordán).

Buxarrais, M. R. y Burget, M. (Coord.) Aprender

a ser. Por una pedagogía de la interioridad

(Gema Pilar Sáez Suanes). Musaio, M. Realizzo

me stesso. Educare i giovani alla ricerca delle possibilità (Carmen Urpí Guercia).

Pérez-Pérez, C. Educación en valores para la ciudadanía. Estrategias y técnicas de aprendizaje (José L. González-Geraldo).

\section{Informaciones}

Conferencia TEPE 2018 sobre «Reclutamiento y educación de los mejores docentes: política, profesionalismo y pedagogíay. I Congreso Internacional sobre «Educación del Carácter en Latinoamérica: Retos y Oportunidades». IX Congreso Internacional de Psicología y Educación (CIPE) sobre «Psicología, Educación y Neurociencias. Construyendo puentes para el desarrollo humano». V Congreso Internacional EDO (CIEDO) sobre «Liderazgo y gestión del talento en las organizaciones».

157 Una visita a la hemeroteca (Eva Ramírez Carpeño). Una visita a la red (David Reyero).

\section{Instrucciones para los autores}

Instructions for authors 201

ISSN 0034-9461 - Depósito legal: M. 6.020 - 1958 e-ISSN 2174-0909 Rev. esp. pedagog. (Internet) INDUSTRIA GRÁFICA ANZOS, S.L. Fuenlabrada - Madrid 